\title{
Image Processing Applied to Medical Science for the Study of Liver Cancer Using Segmentation in Magnetic Resonance Imaging
}

\author{
Jose Ramon Iglesias Gamarra*, Omaira Luz Tapias Diaz \\ Department of Electronic Engineering, Faculty of Engineering, Popular University of Cesar, Valledupar, Colombia \\ Email address: \\ joseiglesias@unicesar.edu.co (J. R. I. Gamarra),omairatapias@unicesar.edu.co (O. L. T. Diaz) \\ ${ }^{*}$ Corresponding author
}

\section{To cite this article:}

Jose Ramon Iglesias Gamarra, Omaira Luz Tapias Diaz. Image Processing Applied to Medical Science for the Study of Liver Cancer Using Segmentation in Magnetic Resonance Imaging. International Journal of Information and Communication Sciences.

Vol. 5, No. 1, 2020, pp. 1-4. doi: 10.11648/j.ijics.20200501.12

Received: July 17, 2019; Accepted: August 13, 2019; Published: May 28, 2020

\begin{abstract}
The objective to develop some algorithms with new techniques of image processing for the automatic segmentation of the liver using magnetic resonance images. The methodology is based in a descriptive description was proposed that allows to combine the information of multiple channels using statistical models that have as a central point the multivariate and multisequence gaussian distribution. In this way, we will approach the spatial distribution having as a central point the intensity values in the different sequences and, therefore, we will be able to capture the variability of the data in each sequence at that moment. The results are based on the segmentation references and the proposed evaluation metrics to be able to validate the development different methods for segmentation were applied as inputs and what was obtained as a result of the segmentation originated a group of images that correspond to each of the cuts that have maximum resolution in the obtained sequences. All the images obtained here including the segmentation referred to, must be binary and their pixels must be marked with 1 (liver) or 0 (without liver). In conclusions the segmentation method that we propose here will consist of an active contour modeling in $2 \mathrm{D}$ and $3 \mathrm{D}$ and that will be developed in images that will be produced based on a new developed descriptor, having as an important point to minimize the image with an Approximation that is dual to the variational problem which should give us good results in the segmentation process.
\end{abstract}

Keywords: Segmentation, Magnetic Resonance (MRI), Image, Metrics, Processing

\section{Introduction}

The treatment of images with application to medicine has become in recent years as an important support for medical diagnosis. By using advanced image reconstruction technologies, we have the possibility to identify different organs and tissues and at the same time obtain data that will help us to characterize and total cancer pathologies.

By studies, we know that liver cancer has a great incidence in the cause of deaths in the world. The current diagnosis is an invasive procedure, with new technologies that we could replace with non-invasive procedures to carry out a reliable planning of the intervention. At the Universidad Popular del Cesar we work on research projects to experiment with new methods and efficiently. Tools such as magnetic resonance images perform an optimal location and thus are able to properly segment the organ of interest and its pathologies through image processing.

To obtain results it is necessary to process the images. The image processing gathers some of the following sequences: it emphasizes and improves the quality of the image, extracts characteristics, segments, quantifies and classifies. In medical images we need operations that can be registered to work with some images of the same physical region which poses the following challenges for research:

1) Pre-processing of the image: the extraction of information from any image becomes a complicated and laborious task. To improve the quality of an image, we must initiate several problems, such as the elimination of noise, the increase of contrast, the 
emphasis on the edges and the most relevant structures, detect points of interest, have uniform illumination, eliminate acquisition artifacts., etc.

2) Extraction of characteristics: here we must understand the set of techniques to identify and extract characteristics and some descriptors that are relevant in the image, which will allow us to represent some differences between zones. These characteristics will depend on gray levels, the histogram itself, its edges and the texture of neighboring pixels.

3) Segmentation of the image: it allows us to separate some elements of an image. It consists in dividing or separating some similar attributes in regions. The segmentation makes a subdivision of an image in the different parts that compose it. The importance of the subdivisions depends on the problem to be solved, so the segmentation must stop when the objects of interest have been isolated.

4) Classification of the image: this technique allows us, from a set of data that we obtain from the segmentation process in the defined region, to assign that region to a group or another, this process is called a class. When assigned to a class, in the case of a medical image, it could be the identification of an injury or type of tissue. But in addition, we could add measurement operations and some quantification in that image to obtain better data, which are valuable in the diagnosis, and better plan the tasks of the surgery and the subsequent treatment.

To interpret an image, it is necessary to specify the identification of the parts that make up what we call segmentation [1-6], but for a segmentation method to succeed, the appropriate choice of descriptors and image characteristics must be the most important step.

When analyzing the problem, some descriptors and characteristics will be more important than others because of the information they can provide.

The descriptors are the most widespread [7-8] and in the treatment of images they are widely used, which leads us to a great use in the treatment of medical images and specifically in liver segmentation, which is the work that concerns us.

In image processing, the descriptor is a feature that helps us describe the visual content of that image. All descriptors that provide basic data at the pixel level are often referred to as low level and represent color characteristics, shape texture and gradient. These values obtained for these descriptors of these images are the entries for the segmentation or classification methods, which locate and identify an element that belongs to a specific class.

The descriptors are often combined with different techniques of artificial intelligence, thus providing semantic information [9-12], which allows us to cover the concept of semantic computation. In this work we will only analyze the descriptors that are used in the treatment of images to analyze and characterize some structures and, therefore, guide them for the realization of liver segmentation.

\section{Method}

For this, the segmentation method that we are developing will allow a fast and accurate segmentation. The method is being developed in $2 \mathrm{D}$ and $3 \mathrm{D}$ and is being validated by means of database images, the second step is to perform it with real diagnoses, and thus be able to compare the results with respect to a segmentation process as a reference source [13-16] with the support of expert radiologists.

Grays levels are the natural descriptors of a monochromatic image. Although the color is represented in its most extended form by 3 matrices or channels: red, green and blue (Red Green Blue (RGB)), each of these matrices is a monochromatic plane. This plane, which is a gray scale, has a number of possible values for each point in the image that we define by the depth of the pixel, that is, by the number of bits that represent a pixel, this is what is called resolution in gray levels Therefore, an image with an 8-bit pixel can have 256 (28) different gray values, while an image with a 12-bit pixel depth can have 4,096 (212) different gray levels. The deeper the pixel is, the more accurate the image is for the characterization of the elements, so that we can appreciate the soft nuances that cause the differences in the elements that are present in the image. As an example, we show below an abdominal image where the information is represented correctly with a 12-bit pixel (Figure 1) and the same information represented with an 8-bit pixel (Figure 2). It can be seen that in the second case some details are lost.
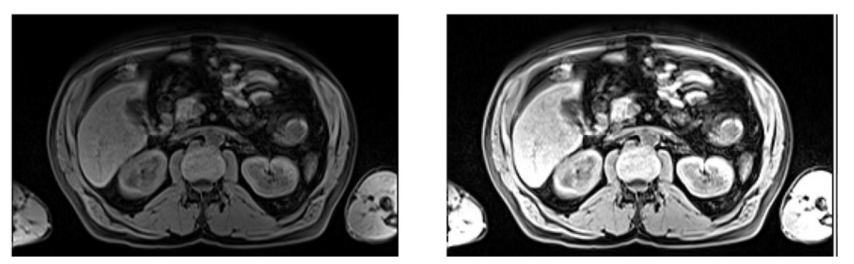

Figure 1. Image with a depth of pixels of 12 bits (left) and 8 bits (right).

Another type of characteristics to describe an object are the shape descriptors. So that we can validate the descriptors and be able to apply them to the characterization, they must comply with the following premises:

a) That can be identified and be reliable: for this we must use different examples of that object that must have similar forms and at the same time be clearly different from another set of elements, so that all the characteristics that we can extract from the images are for represent them.

b) That can be invariant to the translation, rotation and scale: the shape descriptors must meet the above conditions to correctly describe an object. This process joins what we call "related invariance". This affine transformation can be constructed by applying a series of translation, scaling and/or rotation operations. When extracting the characteristics, they must be invariant to those operations, which will allow us in the same space to which it refers to locate objects with different sizes and positions, and thus be able to identify the parameters of said set of elements. 
c) That they are resistant to noise: these descriptors must be very robust in the presence of noise.

d) That they can be invariant to the concealment: to have hidden in a part of the image of an object, the characteristics that describe the form of the rest of the object could not change.

These form descriptors can be represented structurally or statistically but, in any case, they must allow reconstructing the complete object from the characteristic values.

We must bear in mind that images obtained by magnetic resonance can present many problems that make segmentation and automatic characterization difficult [17]. Some of these problems are a low sharpness and, therefore, a low definition of edges, noise and the fact that different fabrics have similar gray levels. Observe in the following image, since we appreciate that the liver (red arrow) presents a level of gray similar to the abdominal wall itself (green arrow) for a certain sequence, which causes us a difficulty in the automatic segmentation process.

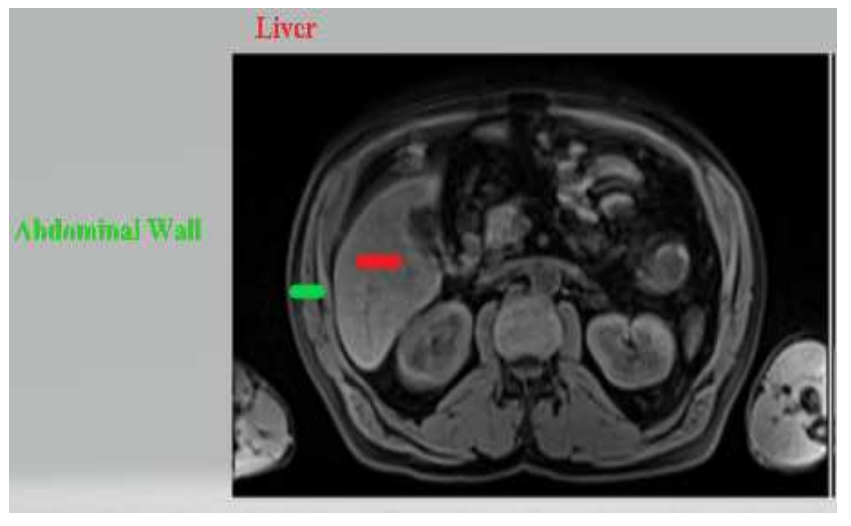

Figure 2. Organs with a similar gray level value in a sequence.

\section{Results}

One of the results we have so far is that we were able to establish references for the segmentation and evaluation metrics and, therefore, validate the development. This leads us to apply each of the proposed segmentation methods in this work and verify the results of said segmentation by means of a set of images that correspond to each of the cuts of the maximum resolution sequence. It is necessary that the images, like the reference segmentation, have to be binary and, because of this process, we had to label their pixels as 1 (liver) or 0 (without liver).

That is why, having segmentation and reference, we have obtained the values of the metrics for each segmentation method.

According to the above, the methods evaluated are:

1. 2D segment with a monosequence entry.

2. 2D segment with a multi-frequency input.

3. 3D segment with a monosequence entry.

4. 3D segment with a multi-frequency input.

We have evaluated the effectiveness of the methods, quantifying the liver segmentation in the images and comparing it with the segmentation of the reference using the evaluation metrics. Each study performed with MRI includes the set of available sequences. Each sequence has approximately 63 images, corresponding to the number of $2 \mathrm{D}$ cuts of said volume or sequence along the axial axis of the human body.

Taking into account the segmented volumes and the reference volumes and after the application of the corresponding expressions, we obtain the values of the metrics for each segmentation method.

Taking into account the proposed methods, we must combine three important elements:

1. The compact descriptor that will generate the distance map.

2. The active contour model element (2D and $3 \mathrm{D})$.

3 . Methods of variation defined as robust minimization techniques (2D and $3 \mathrm{D})$ to perform regularization and smoothing.

We must verify if the union we propose can constitute a segmentation method that can provide accurate results. We should also appreciate if taking the process to an extension to $3 \mathrm{D}$ gives us something of value about $2 \mathrm{D}$ development. We must also evaluate if the compact descriptor that we have proposed and with which we generate the distance map, constitutes a new input that is generated from the inputs of multiple frequencies, which is valid for the handling of multiple information and also allows the inclusion of information with a great complementary value that contributes to better results in the final segmentation.

\section{Conclusions}

In magnetic resonance imaging for liver segmentation is a complicated task. These images give us a lot of information to be able to diagnose, but they are low quality images, they have a lot of noise, artifacts of acquisition and movement, partial volume effect and, therefore, little clarity, which leads us to have little contrast between organs, so the boundary between the many tissues is often confusing. The liver is adjacent to the kidney, intestine, lung, gallbladder or abdominal fat; some of them have gray levels similar to those of the liver. That is why the localization and automatic segmentation of the liver in the MRI images is a difficult task.

This method of segmentation that we propose and that consists of an active contour model in $2 \mathrm{D}$ or $3 \mathrm{D}$ and that is developed in an image that is generated based on a new descriptor, has as an initial parameter a process of minimization of the image through a dual approach to the variational problem, and that gives us good results in segmentation.

This segmentation proposed here reduces the necessary computational calculation time, which decreases the dimension of the problem. That is why all the input sequences are transformed into a single image that represents a map of distances of each of the pixels of the image with respect to a multivariable statistical model of liver and that we had generated before. The constant work of the 
researchers in the accomplishment of the assigned tasks is of great importance that will allow us to reduce the problem if we have several sequences of the same study, which would facilitate the application of techniques of deformable models or active surfaces in this unique entry that contains the information of all the channels.

\section{References}

[1] F. López-Mir, V. Naranjo, J. Angulo, M. Alcañiz, and L. Luna, "Liver segmentation in MRI: A fully automatic method based on stochastic partitions.," Comput. Methods Programs Biomed., vol. 114, no. 1, pp. 11-28, Apr. 2014.

[2] J. Oh, D. R. Martin, and X. Hu, "Partitioned edge-functionscaled region-based active contour (p-ESRAC): Automated liver segmentation in multiphase contrast-enhanced MRI.," Med. Phys., vol. 41, no. 4, p. 041914, Apr. 2014.

[3] Antonidoss, K. P., Kaliyamurthie, (2014). Segmentation from Images Using Adaptive Threshold. Middle-East J. Sci. Res. 20, 479-484. doi: 10.5829/idosi.mejsr.2014. 20. 04. 21037.

[4] P. Bao., L. Zhang., (2003). Noise reduction for magnetic resonance images via adaptive multiscale products thresholding. IEEE Trans. Med. Imaging 22, 1089-99. doi: 10.1109/TMI.2003.816958.

[5] O. Basset., Z. Sun., J. L, Mestas. G. Gimenez., (1993). Texture analysis of ultrasonic images of the prostate by means of coocurrence matrices. Ultrason. Imaging 15, 218-237.

[6] R. Beichel., S. Mitchell., E. Sorantin., (2001). Shape-and appearance-based segmentation of volumetric medical images. ICIP 2, 589-592.

[7] Jia, X., Bian, Z., He, J., Wang, Y., Huang, J., Zeng, D., Ma, J. (2016). Texture-preserved low-dose CT reconstruction using region recognizable patch-priors from previous normal-dose CT images. 2016 IEEE Nuclear Science Symposium, Medical Imaging Conference and Room-Temperature Semiconductor Detector Workshop (NSS/MIC/RTSD). doi: 10.1109/nssmic.2016.8069559.

[8] L. Zhong et al., (2016). Predict CT image from MRI data using KNN-regression with learned local descriptors, Proc.
IEEE 13th Int. Symp. Biomed. Imag., pp. 743-746.

[9] Yang, W., Zhong, L., Chen, Y., Lin, L., Lu, Z., Liu, S., Chen, W. (2018). Predicting CT Image from MRI Data Through Feature Matching With Learned Nonlinear Local Descriptors. IEEE Transactions on Medical Imaging, 37 (4), 977-987. doi: 10.1109/tmi.2018.2790962.

[10] Y. Boykov., G. Funka-Lea., (2006). Graph Cuts and Efficient N-D Image Segmentation. Int. J. Comput. Vis. 70, 109-131. doi: 10.1007/s11263-006-7934-5.

[11] A. Foruzan., C. Yen-Wei., (2013). Segmentation of Liver in Low-Contrast Images Using K-Means Clustering and Geodesic Active Contour Algorithms. IEICE Trans. INF SYST. E96-D, 798-807.

[12] O. Gloger., J. Kühn., A. Stanski., H. Völzke., R. Puls., (2010). A fully automatic three-step liver segmentation method on LDA-based probability maps for multiple contrast MRI images. Magn. Reson. Imaging 28, 882-897. doi: http://dx.doi.org/10.1016/j.mri.2010.03.010.

[13] O. Gloger., K. Toennies., J. P. Kuehn., (2011). Fully Automatic Liver Volumetry Using 3D Level Set Segmentation for Differentiated Liver Tissue Types in Multiple Contrast MR Datasets, in: Heyden, A., Kahl, F. (Eds.), Image Analysis SE48, Lecture Notes in Computer Science. Springer Berlin Heidelberg, pp. 512-523. doi: 10.1007/978-3-642-212277_48.

[14] E. Göçeri., M. N Gürcan., O. Dicle., (2014). Fully automated liver segmentation from SPIR image series. Comput. Biol. Med. 53, 265-278. doi: 10.1016/j.compbiomed.2014.08.009.

[15] H. T. Huynh., I. Karademir., A. Oto., K. Suzuki., (2014). Computerized liver volumetry on MRI by using 3D geodesic active contour segmentation. AJR. Am. J. Roentgenol. 202, 152-9. doi: 10.2214/AJR.13.10812.

[16] A. P. James., B. V. Dasarathy., (2014). Medical image fusion: A survey of the state of the art. Inf. Fusion 19, 4-19. doi: 10.1016/j.inffus.2013.12.002.

[17] F. López-Mir., V. Naranjo., J. Angulo., M. Alcañiz., L. Luna., (2014). Liver segmentation in MRI: A fully automatic method based on stochastic partitions. Comput. Methods Programs Biomed. 114, 11-28. doi: 10.1016/j.cmpb.2013.12.022. 